\title{
Genetic susceptibility to type 2 diabetes and obesity: from genome-wide association studies to rare variants and beyond
}

\author{
Niels Grarup • Camilla H. Sandholt • Torben Hansen • \\ Oluf Pedersen
}

Received: 13 December 2013 / Accepted: 22 April 2014 / Published online: 24 May 2014

(C) Springer-Verlag Berlin Heidelberg 2014

\begin{abstract}
During the past 7 years, genome-wide association studies have shed light on the contribution of common genomic variants to the genetic architecture of type 2 diabetes, obesity and related intermediate phenotypes. The discoveries have firmly established more than 175 genomic loci associated with these phenotypes. Despite the tight correlation between type 2 diabetes and obesity, these conditions do not appear to share a common genetic background, since they have few genetic risk loci in common. The recent genetic discoveries do however highlight specific details of the interplay between the pathogenesis of type 2 diabetes, insulin resistance and obesity. The focus is currently shifting towards investigations of data from targeted array-based genotyping and exome and genome sequencing to study the individual and combined effect of low-frequency and rare variants in metabolic disease. Here we review recent progress as regards the concepts, methodologies and derived outcomes of studies of the genetics of type 2 diabetes and obesity, and discuss avenues to be investigated in the future within this research field.
\end{abstract}

Keywords Adiposity · Body mass index · Genetics . Genome-wide association · Glycaemic traits - Obesity · Review $\cdot$ Sequencing $\cdot$ Type 2 diabetes

N. Grarup $(\bowtie) \cdot$ C. H. Sandholt $\cdot$ T. Hansen $\cdot$ O. Pedersen The Novo Nordisk Foundation Center for Basic Metabolic Research, Faculty of Health and Medical Sciences, University of Copenhagen, DIKU Building, Universitetsparken 1, 2100 Copenhagen Ø, Denmark

e-mail: niels.grarup@sund.ku.dk

T. Hansen

Faculty of Health Sciences, University of Southern Denmark,

Odense, Denmark

\author{
Abbreviations \\ DIAGRAM DIAbetes Genetics Replication And \\ Meta-analysis \\ GIANT Genetic Investigation of ANthropometric \\ Traits \\ GWAS Genome-wide association study \\ MAF Minor allele frequency \\ MAGIC Meta-Analyses of Glucose and Insulin-related \\ traits Consortium \\ SNP Single-nucleotide polymorphism
}

\section{Introduction}

On a global scale, the prevalence of obesity and type 2 diabetes is increasing dramatically, and WHO reports that more than 500 million people are obese [1] and 346 million have diabetes, $90 \%$ of whom have been diagnosed with type 2 diabetes [2]. Lifestyle and environmental factors are crucially important in the development of obesity and type 2 diabetes. Important risk factors for obesity are physical inactivity, excessive energy intake, depression, sleep disorders and low socioeconomic status, while major risk factors for type 2 diabetes include obesity, especially visceral fat deposition, physical inactivity, smoking, male sex, high age, sleep deprivation, urbanisation, low-socioeconomic status and ethnicity [3-6]. In addition, $40-70 \%$ of BMI variation is explained by genetic factors $[7,8]$ and, similarly, the increase in type 2 diabetes risk associated with having a sibling with type 2 diabetes is two- to threefold [9]. Family studies have shown similar heritability estimates of $50-60 \%$ for BMI and type 2 diabetes [10]. Since 2007, an explosion in our knowledge of specific genetic risk factors for obesity, type 2 diabetes and related phenotypes has taken place, mainly brought about by genome-wide association studies (GWASs). Here we review 
recent progress in concepts, methodologies and derived outcomes of studies of the genetics of type 2 diabetes and obesity, and we predict some of the directions this research field could take in the near future.

\section{GWASs to discover the genetic basis of type 2 diabetes and obesity}

Until 2007, genetic mapping of complex diseases such as type 2 diabetes and obesity was primarily achieved by genetic linkage analyses or candidate gene association studies, both of which have implicit shortcomings related to their design, which limit their application. At the same time, study sample sizes were generally too low to reach sufficient statistical power. However, progress in identifying common variants associated with type 2 diabetes and obesity has since been rapid, primarily as a consequence of technological advances in array-based genotyping, which paved the way for GWASs, together with increased sample sizes from international collaborations. These advances have led to the discovery of a wealth of genomic loci convincingly associated with complex metabolic traits [5-7].

\section{Genetics of type 2 diabetes and glucose homeostasis: what is known?}

As of the beginning of 2014, 90 genetic loci have been firmly established as type 2 diabetes risk loci (Fig. 1) [11-25]. The risk variant in the TCF7L2 locus, which was discovered in 2006 by a positional linkage strategy in the Icelandic population [26], remains the most influential common type 2 diabetes variant (allelic OR 1.46) [27]. While GWASs of type 2 diabetes have been highly successful, other type 2 diabetes-associated loci have been identified through studies of quantitative diabetes-related traits. These efforts have discovered 72 loci associated with quantitative traits reflecting glucose homeostasis, i.e. fasting glucose, fasting insulin, $2 \mathrm{~h}$ glucose during an OGTT and $\mathrm{HbA}_{1 \mathrm{c}}[16,28-31]$. Many of these loci are also associated with type 2 diabetes, yet the overlap between loci for these traits is not extensive (Fig. 1). In recently published reports, a mere 13 of 37 variants associated with fasting glucose were also associated with type 2 diabetes at what are considered statistically significant levels for GWASs [13, 29]. These findings indicate that some genetic variants may exert general modifying effects on fasting glucose levels in the population, while others have specific thresholds at which the genetic effect sets in, thereby conferring risk of type 2 diabetes without modifying levels of fasting glucose at the population level.

Genetic variants associated with type 2 diabetes and glucose homeostasis can shed light on the relationship between genetically induced defects in insulin secretion and insulin action in the pathogenesis of type 2 diabetes. Most of the genetic variants found in the first GWASs were demonstrated to primarily cause a decrease in glucose-stimulated insulin response [32,33]. A more detailed picture of beta cell pathogenesis is now emerging showing the specific impact of individual risk variants through studies of more exact
Fig. 1 Venn diagram of intersection between loci associated at genome-wide significance with type 2 diabetes, measures of adiposity and glucose homeostasis. Genome-wide significant associations for six metabolic traits are shown. Gene symbols shown in the plot are by convention the closest gene and not necessarily the functional gene




physiological phenotypes and functional molecular genetic studies. Specific defects in glucagon-like peptide 1-stimulated insulin secretion, glucose-stimulated insulin secretion, insulin exocytosis, insulin granule docking or post-transcriptional processing of insulin have been demonstrated to be associated with different variants, supporting the notion that a range of biological processes are involved in the pathogenesis of type 2 diabetes [33-38]. As for other epidemiological studies of insulin secretion, these efforts are impeded by the difficulty of accurately quantifying insulin secretion in an epidemiological setting. The insulin response is generally assessed as the secretion of insulin in response to a number of different secretagogues, all of which provide different physiological information [39]. Therefore, studies of this trait tend to be small and statistically underpowered to detect the modest effect of single SNPs.

In the first GWASs of type 2 diabetes and quantitative glucose homeostasis traits, few variants were shown to have an effect on insulin sensitivity [16, 40]. Of interest, the number of SNPs associated with insulin sensitivity has recently increased as a result of larger samples sizes, by the inclusion of concomitant levels of obesity as a covariate in regression models and by implementation of a joint test investigating the main effect while allowing for an interaction effect [29, 30]. Thus, many primary genetic defects in insulin sensitivity may not be mediated by levels of obesity.

\section{Genetics of obesity and measures of body fat distribution}

The discovery of variants associated with measures of adiposity through GWAS follows much the same story as identification of the type 2 diabetes risk variants. The discoveries have predominately involved individual studies and metaanalyses using BMI as a quantitative measure of adiposity. The first studies, which included relatively few individuals, identified two loci, FTO and MC4R [41, 42]. The necessity to increase statistical power led to larger studies that included $\sim 32,000$ individuals, and as a result an increased number of loci were identified $[43,44]$. The largest meta-analysis performed using BMI as a measure of obesity included $\sim 250,000$ individuals and increased the number of identified BMI loci to 32 [45]. FTO, the first GWAS-identified obesity locus, remains the one with the largest effect, imposing an allelic $0.39 \mathrm{~kg} / \mathrm{m}^{2}$ increase in BMI [41].

In parallel with the studies of BMI, a crude measure of overall adiposity, GWASs of quantitative measures attempting to capture abdominal obesity and specific elements of fat distribution, such as waist circumference and WHR, have also been performed. Studies including up to $\sim 77,000$ individuals have identified 19 loci associating with measures of body composition [46-48], the vast majority associating with BMI-adjusted WHR [48]. Since most of the body composition loci have been identified by analysing WHR adjusted for BMI, no overlap exists between these and the 39 BMI loci (Fig. 1).

Within the obesity-GWAS framework, case-control approaches in children, adolescents and adults have also been widely used [49-53]. The earliest studies were relatively small and substantial overlap with both BMI and body composition loci were detected. Two recent studies have increased the number of loci to 19 , identifying 15 non-overlapping loci associated with clinical obesity among children and adults $[54,55]$. There are several possible explanations for the partial genetic overlap of BMI and clinical definitions of obesity. While GWASs of BMI have focused on SNPs associated with mean BMI in populations, studies indicate that effects for many loci are not uniform across the BMI distribution. In studies of childhood BMI, the effect of several loci, including FTO, was stronger in the upper tail of the BMI distribution [56]. Along the same lines, a recent study showed that FTO genotype, which has been shown to be convincingly associated with mean BMI, is associated with variance in BMI [57], and since variance in BMI increases with BMI, this observation may explain the association of FTO with both mean BMI and severe clinical obesity. In addition, these studies point to interaction between genotype and measured or unmeasured environmental factors. Overall, GWASs have to date successfully identified more than 80 different loci associated with adiposity phenotypes. These results point to the existence of aetiologically distinct subsets of extreme phenotypes.

\section{Transferability of genetic loci across ancestry groups}

Although thus far the majority of GWASs have been performed on European individuals, a number of important studies of other ethnicities are emerging. These studies have reported novel loci such as $K C N Q 1$ and $C 2 C D 4 A$ associated with type 2 diabetes in Japanese individuals [17, 58, 59] and a number of loci for type 2 diabetes in East Asians [20, 22]. For obesity, studies have identified risk variants in PCSK1, GP2 and GALNT10 loci in Asian or African populations [60, 61]. Of interest, studies comparing associations in individuals of different ethnicities can shed light on the shared genetic vulnerability across ethnic groups and possibly add to finemapping efforts in associated loci. For type 2 diabetes, studies have found directionally consistent effects for known loci across ancestry groups $[62,63]$. Comparative studies across ancestries in a recent GWAS of four ancestry groups showed that the effects of the many common variants not reaching statistical significance at a genome-wide level are homogenous across ancestry groups, and a trans-ancestry metaanalysis revealed seven novel genome-wide significant loci [25]. In addition, the study showed that fine-mapping associated loci can be improved by taking advantage of ancestry 
differences in linkage disequilibrium. Similarly, studies of obesity have shown highly comparable effects of common variants across major ancestry groups, strongly supporting shared common BMI and obesity loci across populations $[60,61,64]$, although ancestry-specific loci have also been shown, such as KLHL32 in Africans and KLF9 in Asians $[61,64]$.

\section{Glossary \\ 1000 Genomes Project The 1000 Genomes Project,} launched in January 2008, is an international research effort to establish a detailed catalogue of human genetic variation. Scientists planned to sequence the genomes of 2,500 participants from a number of different ethnic groups

Allele One of a number of alternative forms of the same gene or same genetic locus

De novo mutation An alteration in a gene that is present for the first time in one family member as a result of a mutation in a germ cell (egg or sperm) of one of the parents or in the fertilised egg itself

Epistasis When the effect of one gene depends on the presence of one or more 'modifier genes' (genetic background). Also referred to as gene-gene interaction

Exome The protein coding part of the human genome. The exome of the human genome consists of roughly 180,000 exons, constituting about $1 \%$ of the total genome, or about 30 megabases of DNA

Heritability The proportion of phenotypic variation of a trait that is due to underlying genetic variation

Imputation In genetics, imputation refers to the statistical inference of unobserved genotypes. It is achieved by using known haplotypes in a reference population, such as the 1000 Genomes Project, thereby allowing non-genotyped genetic variants to be tested for association with a trait of interest

Linkage disequilibrium A non-random association between alleles at different loci

Minor allele frequency Ranging from $0 \%$ to $50 \%$, this is the proportion of alleles at a locus that contain the less frequent allele.

Private variants Variants restricted to probands and immediate relatives

Sequencing depth In DNA sequencing, depth refers to the number of times a nucleotide is read during the sequencing process. Deep sequencing indicates that the depth of the process is many times larger than the length of the sequence under study
Common features of the genetic associations with type 2 diabetes, glycaemia and obesity

Implicit in the initial design, GWAS-identified variants in type 2 diabetes, glycaemia and obesity are common (minor allele frequency $[\mathrm{MAF}]>5 \%$ in the population). Risk variants exert modest effect sizes on disease risk and variation in phenotype, and for the majority of loci, the causative variant and gene is unknown. For the majority of loci, the most strongly associated variant is not a coding variant but instead resides in an intron or in a non-coding sequence between genes. In addition, high correlation (i.e. linkage disequilibrium) between physically closely located markers makes it difficult to prove causality for associated variants. However, the causative variant and the molecular mechanism of action have been identified for some loci. In the GCKR locus an intronic variant was shown to associate with type 2 diabetes and fasting glucose [65], while subsequent studies detected an amino acid-changing variant (GCKR p.P446L) that was demonstrated to be the causative variant influencing hepatic glucose uptake [66, 67]. Similarly, follow-up studies of TCF7L2 have shown that the originally identified intronic rs7903146 variant is probably the causative SNP, which presumably regulates expression of alternative $T C F 7 L 2$ isoforms in several target tissues [68-71]. Many of the loci do not contain genes with known biological relevance to obesity or type 2 diabetes, providing an opportunity for novel biological investigations. Finally, common risk variants have been found in a number of genes known to be mutated in monogenic subsets of non-autoimmune diabetes (GCK, HNF1A, HNF1B, HNF4A, PPARG, KCNJ11, GLIS3 and WFS1) [11, 12, 16, 72-80] or obesity (MC4R, POMC, LEPR, BDNF, SH2B1, PCSK1 and NTRK2) [42, 45, 81-87].

\section{Genetic overlap of obesity and type 2 diabetes: epidemiological vs genetic correlation}

Although type 2 diabetes and obesity are highly interrelated from both epidemiological and pathophysiological viewpoints, the shared genetic aetiology imposed by hitherto identified common variants is limited (Fig. 1). Of 90 loci associated with type 2 diabetes and 56 loci associated with standard measures of adiposity, merely five loci are shared (FTO, MC4R, ADAMTS9, GRB14/COBLL1 and QPCTL/GIPR). Furthermore, at two of these loci (ADAMTS9 and GRB14/ $C O B L L 1$ ), different and only partially correlated genetic variants are responsible for the associations, which brings into question whether they share functional disease mechanisms $[11,13,14,48]$. There are many possible reasons for this apparent lack of genetic overlap. For example, the associations between genetic loci and traits shown in Fig. 1 are for associations at genome-wide statistical significance and 
therefore do not include shared associations below the level of significance, which may still be genuine.

Some light can be shed on the relationship between SNPs associated with type 2 diabetes or glycaemic traits and associations with measures of obesity using online large-scale databases of GWAS results. Figures 2 and 3 illustrate the correlated effects of SNPs associated with type 2 diabetes or BMI for a specific set of GWAS SNPs for a particular trait, comparing the effect on the primary trait with other correlated metabolic traits. For SNPs primarily associated with BMI, there seems to be a positive correlation between the effect size on BMI and the effect of the same SNP on type 2 diabetes (Fig. 2a). This finding indicates that the major reason why so few BMI-associated SNPs have been shown to associate with type 2 diabetes at genome-wide significance is a lack of statistical power to detect the minute derived type 2 diabetes risk increments imposed by BMI-associated variants. Similarly, there is a positive correlation between the effect of BMI SNPs on BMI and on the diabetes-related quantitative traits, fasting glucose and fasting insulin (Fig. 2b, c). In contrast, when looking at the effect sizes on BMI of SNPs associated with type 2 diabetes, there is no obvious correlation between effects; rather, it seems that most of the type 2 diabetesassociated variants have no impact on BMI per se (Fig. 3a). Similar observations are evident when comparing the effects of type 2 diabetes-associated SNPs on type 2 diabetes and
WHR (Fig. 3b). A general effect on fasting glucose is seen for type 2 diabetes variants, yet no correlation with the effect on fasting insulin is evident (Fig. 3c, d). These observations are in line with the finding that most common type 2 diabetes risk variants have an intermediate impact on the ability to secrete appropriate amounts of insulin [33].

The findings described above are compatible with correlations between type 2 and obesity in epidemiological studies and the current view that obesity is one of the causes of type 2 diabetes. These relationships are illustrated by the effect of variation in FTO on type 2 diabetes. While initial findings showed an association with BMI and with type 2 diabetes mediated by obesity $[41,88,89]$, other studies have pointed to an effect on type 2 diabetes that is independent of adiposity $[90,91]$. While this may be a true adiposity-independent effect, it may also be caused by an inability to properly correct for adiposity by the use of BMI as covariate or by multiple functional mechanisms for variation in the FTO locus [92]. Nevertheless, correlation between two heritable traits in epidemiological studies does not necessarily lead to the conclusion that underlying genetic determinants between the two traits are similar. A critical feature is the amount of additive genetic variation shared between two traits, which can be estimated from family or twin studies and is expressed by a genetic correlation coefficient. If two epidemiologically correlated traits are partly heritable, the genetic correlation may
Fig. 2 Correlation of effects of BMI-associated loci on BMI in relation to effects on type 2 diabetes and metabolic traits. Each dot shows the effect of a BMI-associated variant on BMI in relation to the effect on (a) type 2 diabetes, (b) fasting glucose, (c) fasting insulin and (d) WHR adjusted for BMI. Dots coloured red indicate an association $(p<0.005)$ with the trait on the

y-axis. Effect sizes and $p$ values were obtained from the largest available GWAS for the trait: type 2 diabetes $\left(n_{\text {cases }}=12,171\right.$ and $\left.n_{\text {controls }}=56,862\right)$ from the DIAGRAM Consortium (http:// diagram-consortium.org/) [13]; fasting glucose $(n=58,074)$ and fasting insulin $(n=51,750)$ from MAGIC (http://www. magicinvestigators.org/) [30]; and BMI $(n=123,912)$ and WHR $(n=77,149)$ from the GIANT consortium [45, 48]. Not all variants found in GWASs are included in these graphs

Fig. 3 Correlation of effects of type 2 diabetes-associated loci on type 2 diabetes in relation to effects on BMI and metabolic traits. Each dot shows the effect of a type 2 diabetes-associated variant on type 2 diabetes in relation to the effect on (a) BMI, (b) WHR adjusted for BMI, (c) fasting glucose and (d) fasting insulin. Dots coloured red indicate an association $(p<0.005)$ with the trait on the y-axis. Effect sizes and $p$ values were obtained from the largest available genome-wide association study for the trait: type 2 diabetes $\left(n_{\text {cases }}=12,171\right.$ and $n_{\text {controls }}=$ 56,862 ) from the DIAGRAM consortium (http://diagramconsortium.org/) [13]; fasting glucose $(n=58,074)$ and fasting insulin $(n=51,750)$ from MAGIC (http://www.magicinvestigators. org/) [30]; and BMI $(n=123,912)$ and WHR $(n=77,149)$ from the GIANT consortium [45, 48]. Not all variants found in GWASs are included in these graphs a

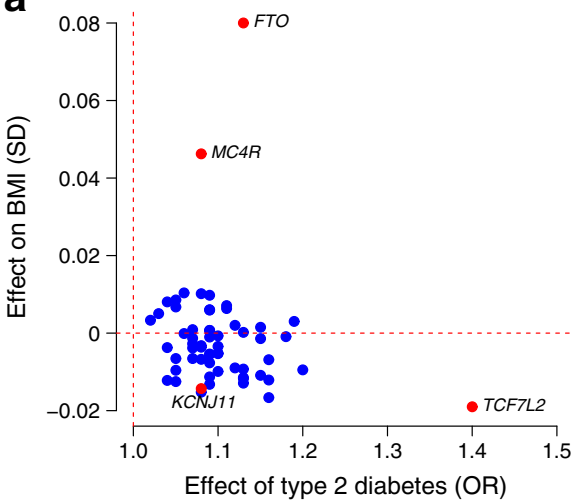

C



b

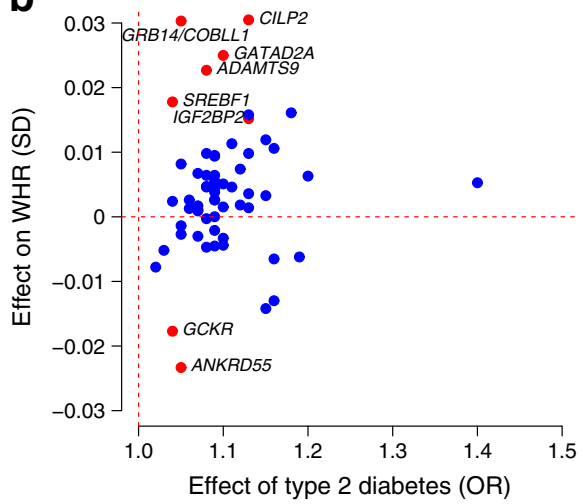

d



still be modest or absent, indicating that different specific genetic factors are contributing to variation in the traits. Even genetically correlated traits may only share a minor fraction of quantitative trait loci [93]. Two large studies have tried to model the genetic correlation between type 2 diabetes and BMI by studying twin populations of more than 20,000 twins of Finnish and Swedish origin with long-term follow-up. Both studies found a high heritability of BMI and type 2 diabetes, yet the genetic correlation between BMI and type 2 diabetes was estimated to be $\sim 40-45 \%$, indicating that around one-fifth of the covariance of BMI and type 2 diabetes is due to shared genetic influences [94, 95]. Interestingly, these findings are consistent with discoveries from GWASs (Fig. 1).

\section{GRB 14/COBLL1: a genetic locus with a pleiotropic effect on the metabolic syndrome}

Comparisons of effect sizes for loci associated with adiposity measures and type 2 diabetes indicate that not all of the more than 175 loci associated with common metabolic phenotypes display pleiotropic metabolic effects. GRB14/COBLL1 is a locus that has been shown to associate with a range of traits. Initially the major allele of rs 10195252 near GRB14 was shown to associate with increased WHR [48]. Other studies have shown associations of the WHR-increasing allele or moderately correlated alleles with increased risk of type 2 diabetes [13, 14, 20], increased fasting insulin [29, 30], decreased HDL-cholesterol and increased triacylglycerol concentration [96], even after adjusting for obesity measures [97]. These associations are in concordance with a general pleiotropic metabolic risk profile affecting many of components of the metabolic syndrome in a clinically unfavourable direction. The nearest genes to the associated SNPs are GRB14 and $C O B L L 1$. Initial reports showed that the risk variant located upstream of GRB14 was associated with mRNA expression of GRB14 in both subcutaneous and omental fat tissues [48]. These data are supported by evidence from studies of Grb14deficient mice showing improved glucose homeostasis despite lower circulating insulin levels and enhanced insulin signalling in liver and skeletal muscle [98]. This locus therefore seems to be an example of genetic risk variants showing metabolic pleiotropic effects.

\section{Metabolically healthy vs metabolically unhealthy associations for obesity-associated variants}

In general, alleles that associate with increasing BMI or WHR would also be expected to increase other metabolic risk variables such as cholesterol, triacylglycerol, glucose and insulin levels and risk of type 2 diabetes, thereby showing a general 
metabolic adverse profile. These expectations have been fulfilled for a genetic risk score constructed from 24 obesityassociated variants, which was strongly associated with insulin resistance and with risk of type 2 diabetes; yet, both associations were abolished after adjustment for BMI [99]. While some loci display such profiles, others have either limited pleiotropic effects or even show paradoxical associations. As described above, GRB14/COBLL1 is an example of an obesity-associated locus with pleiotropic effects on a range of phenotypes related to type 2 diabetes where all associations follow the expected metabolically unhealthy profile $[13,14$, 29, 30, 96, 97]. Similarly, in the FTO, MC4R and GIPR loci, the alleles shown to increase obesity susceptibility are simultaneously associated with increased risk of type 2 diabetes [13, 41, 42, 45] (Fig. 1). Other obesity-associated variants exert distinct effects on other metabolic traits.

In the IRSI locus, a variant located upstream of IRSI was reported to be associated with increased risk of type 2 diabetes and decreased insulin sensitivity [40]. However, in a GWAS of body fat percentage the same locus emerged showing decreased body fat percentage for a perfectly correlated variant [100]. Interestingly, the allele associated with decreasing body fat percentage was also associated with decreased IRSI expression and with an impaired metabolic profile, including an increased visceral fat:subcutaneous fat ratio, insulin resistance, dyslipidaemia, risk of type 2 diabetes and coronary artery disease and decreased adiponectin levels [100]. These findings establish IRS1 as an obesity-associated locus concurrently displaying a metabolically healthy profile, underlining the difficulties involved in accurately assessing specific components of obesity in an epidemiological setting and the subtle differences in the function and regulation of distinct adipose compartments.

A recent meta-analysis of data on up to $\sim 37,000$ individuals systematically evaluated metabolic pleiotropic associations for BMI- and WHR-associated variants [97]. Analysis of individual variants revealed that some were associated with a metabolically unhealthy profile whereas others displayed more complex associations [97]. Genetic risk scores, generated by adding the number of risk alleles for each individual, indicated that a high score based on WHR-associated variants had an adverse effect on serum lipid levels even after adjusting for adiposity. Interestingly, analyses of the genetic risk score based on BMI-associated alleles showed a generally metabolically unhealthy profile, which was abolished after adjustment for BMI, yet analyses adjusted for BMI revealed paradoxical associations with decreased plasma glucose at $2 \mathrm{~h}$ during an OGTT and with decreased systolic and diastolic BP for increasing number of BMI-increasing alleles [97]. Up-coming studies with even larger sample sizes will explore relationships between associations of specific loci and a range of metabolic traits and will probably elucidate further distinct mechanisms in adipose tissue function and physiological relationships between metabolic variables. Thus, knowledge of specific genetic variation associated with metabolic complications in obesity may in theory divide obese people into metabolically healthy and unhealthy subgroups. Yet, the evidence for the existence of so-called healthy obese individuals is conflicting, and recent studies question the importance of such groupings [101, 102].

\section{Genetic architecture of type 2 diabetes: rare variants in a common disease?}

The past 7 years of genetic discoveries brought about by the GWAS approach have meant a giant leap for genetic research of complex traits, with more than 175 genetic loci shown to associate with metabolic traits. Yet, the major part of the genetic predisposition to these phenotypes remains unaccounted for since the proportion of variance explained by genetic risk variants discovered to date is $<10 \%$ for type 2 diabetes [13] and $<2 \%$ for BMI [45]. There has been much focus on this missing heritability [103], which has revived the discussion of the overall composition of the genetic susceptibility, the genetic architecture, of type 2 diabetes, obesity and similar complex diseases. Hence, the 'common variant-common disease hypothesis' [104, 105], stating that the predisposition to common diseases stems from a moderate number of common variants, has largely been refuted. On the other hand, the 'rare variant hypothesis' [106, 107], which suggests that rare alleles with large effects are the primary drivers of common disease, has received renewed attention. Rare variants are common in the sense that they severely outnumber common variants in the human genome [108, 109]. Furthermore, evolutionary theory predicts that disease alleles should be rare, since even a minute fitness reduction will keep allele frequencies low as a result of negative selection [110]. In contrast, the 'thrifty genotype hypothesis' states that genetic variation advantageous during human evolution might now confer risk of disease owing to changes in living conditions and environmental exposures. Positive selection of genotypes increasing energy storage would drive such variations to high frequency [111]. However, a recent study of 65 common type 2 diabetes risk variants did not support this hypothesis [112]. Several examples of the association of rare variants with common disease have been demonstrated. For example, studies of candidate genes in fasting HDL-cholesterol [113] or obesity [114-116], GWAS loci in inflammatory bowel disease [117] and the GWAS-detected MTNR1B in type 2 diabetes [118] have all demonstrated rare variants with individual or combined impact on risk of common, complex diseases. Alternative models describing the genetic architecture of common diseases have been suggested. In the 'infinitesimal model', genetic susceptibility is composed of thousands of common variants, each with minute effects on disease risk [119]. 
Current findings from GWASs seem to be somewhat consistent with this model. For example, the current count of common variants regulating circulating fasting lipids is 157 [120], and estimations from the distribution of association signals in GWAS data of type 2 diabetes point to the existence of more than 400 common, low-effect loci [13]. Furthermore, for many traits, GWASs have shown that increasing sample size leads to seemingly endless discoveries of variants with minor effects $[13,45,120]$, reflecting the fact that the loci detected to date are merely the highest effect sizes according to a curve of distribution.

Each of these models describes extreme scenarios concerning the genetic architecture of common diseases; however, they are not mutually exclusive. Parts of all these hypotheses may contribute to the genetic architecture of type 2 diabetes and other common diseases, raising the possibility of a complex composite model in which context-dependent variation of a range of frequencies, individually or in specific combinations, contributes to genetic susceptibility. The existence of such a composite model is supported by a recently reported simulation analysis, the investigators of which concluded that extreme models are unlikely, yet the simulation data were consistent with many models, including those in which rare variants explain either little or most of type 2 diabetes heritability [121].

In addition, influences other than main effects of SNPs may explain parts of the susceptibility for metabolic disease. Preliminary evidence supports the role of copy number variations and gene-environment interactions in obesity and type 2 diabetes, as solid findings have been reported [122-124]. Furthermore, specific parent-of-origin effects have been shown for type 2 diabetes [125], and future large-scale studies may reveal gene-gene interactions. Of interest, the heritability of type 2 diabetes and obesity may have been overestimated because of failure to account for epistasis among loci, and parts of the missing heritability may be dissolved if epistatic effects exist [126].

\section{Strategies for identifying disease-associated variants across the allele frequency spectrum}

New approaches are currently being used in the search for the determinants of genetic susceptibility to metabolic diseases and other complex traits. Nucleotide sequencing by high-throughput methods, commonly referred to as next-generation sequencing [108, 127], is being extensively used in direct association studies as improved reference panels for imputation of common and lowfrequency variation or to guide the content of novel genotyping arrays (Fig. 4).

GWAS in the current setting with HapMap (http:/hapmap. ncbi.nlm.nih.gov/) or 1000 Genomes (www.1000genomes.org/) imputation has proved to be a powerful tool for finding common disease-associated variants, and increasing the quality of the reference panels used in imputation will increase the genomic coverage of common variants towards an upper limit. In European populations, the current versions of imputation-based association studies are effectively limited to capture variants at an allele frequency above $\sim 1 \%[128$, 129], yet in other populations this approach can also shed light on rare variants. As such, DeCODE Genetics has published a series of reports on the role of rare variants in complex diseases and traits in the Icelandic population based on chip genotyping in large numbers, whole genome sequencing in a subset combined with long-range phasing and genealogybased imputation $[15,127,130]$.

Low-frequency variants (MAF $0.5-5 \%$ ) are probably best studied in a design similar to GWASs (i.e. single SNP
Fig. 4 Suggested study and analysis designs of genetic studies of variants across the allele frequency spectrum. The $\mathrm{x}$-axis designates genetic variation across the allele frequency spectrum. (a) shows suggested study and analytical designs, (b) shows the recommended data generation strategies and technologies




association analyses) on data obtained from either genomewide genotyping and imputation or from targeted array-based genotyping informed by sequencing studies (Fig. 4). A number of studies focused on the detection of lowfrequency variants through whole exome sequencing and/or targeted genotyping have been reported [14, 131-133]. Up to now, such studies have had limited success, probably as a consequence of limited sample size and statistical power. For rare variants (MAF $<0.5 \%$ ), a simple extension of the GWAS paradigm is probably not sufficient since single variant tests are underpowered for the detection of such variants. To circumvent the lack of statistical power of single marker tests, several collapsing or burden methods that simultaneously analyse multiple rare variants are applied [134, 135]. These methods test the cumulative effect of multiple rare variants in a genomic unit, such as a gene or pathway. So far, few whole exome or genome sequencing studies applying such methods to the investigation of rare variants in complex diseases have been published. In a recent study of the exomes of 2,000 individuals, we were unsuccessful in our aim of discovering genes harbouring rare variants associated with type 2 diabetes [136]; however, larger sample sizes are needed to make general inferences of the importance of rare variants in the genetic architecture of metabolic traits. Ongoing work in international consortia will probably lead us closer to answering this question, yet it may be expected that large numbers of novel loci will not be identified until massive sample sizes are achieved. Of importance, the best grouping of coding rare variants in burden tests is as yet undecided. Most published studies have included disruptive and missense variants below a certain frequency threshold, but including many neutral missense variants in burden tests will decrease statistical power [126]. However, dividing rare missense variants based on functionality is not a trivial task. In silico functional prediction methods are not accurate, although studies indicate that the best of these methods can be valuable in filtering rare missense variants [126]. Future integration of high-throughput functional genetic investigations may further improve studies of rare coding variants. Extending such paradigms to noncoding regions is a great challenge since the functional characterisation and prediction of non-coding variation is less advanced.

\section{Family studies revisited in genetic studies of type 2 diabetes?}

Single variant and burden analyses in large-scale exome or genome data will enable detection of rare variants, which segregate in the population. Variations that are specific to the individual or to a single family (so-called private variants) are not present in the general population at any reasonable frequency but constitute a large fraction of all variation and are very difficult to disentangle. From the lessons learned from the study of Mendelian diseases it is plausible that private or family-specific variants with a relatively high impact on disease risk exist; however, to date, the importance of such variants in genetic architecture remains elusive. If such variants cluster within specific genes they may be detectable in large-scale sequencing studies of unrelated individuals, although locus heterogeneity will severely impede detection. Alternatively, private variations may be studied by deep sequencing in extended, multi-generational families, drawing on advantages of accurate family-based imputation of variation and the fact that more observations of the rare variations can be made in large families [137]. However, since both obesity and, especially, type 2 diabetes are rather late-onset diseases, recruiting such families is challenging. While previous genome-wide linkage studies were not particularly successful in mapping disease genes, it is evident that these studies were statistically underpowered in the presence of genetic heterogeneity and that variants associated with less than a fourfold increase in the risk of disease are expected to generate inconsistent linkage results [138]. These observations make it likely that undiscovered private or familyspecific variants with moderate to high impact segregate in families. Yet, the effect of such variants is extremely difficult to prove with statistical confidence.

\section{De novo mutations - a novel risk factor in metabolic disease?}

Family studies make it possible to identify de novo mutations and seek to associate them with disease (Fig. 4). To detect de novo mutations, sequencing data in trios are needed to distinguish rare family-specific mutations from de novo mutations, and association to disease can be done in extended pedigrees or in phenotypically discordant sibling pairs. A number of reports have demonstrated that de novo copy number variations and point mutations are rare but high-impact risk factors in autism spectrum disorders [139, 140]. Yet, the influence of such variants on the risk of type 2 diabetes and metabolic disease is currently unknown. However, upcoming exome or genome sequencing studies, several of which have a familybased design, may lead to discoveries in this research area. Of interest, the diversity in the mutation rate is influenced by the age of the father at conception of the child [141]. Furthermore, de novo mutations have been shown to be a frequent cause of permanent neonatal diabetes and de novo mutations in $H N F 1 B$ is a cause of MODY5 [77, 142]; however, discovery of de novo risk variants is most likely primarily possible for 
variants, with large effects on disease risk placing them under selective pressure [126].

\section{Summary}

- GWASs have established more than 175 genetic risk variants for human type 2 diabetes, glycaemia and adiposity

- The shared genetic aetiology of type 2 diabetes and obesity discovered in GWASs is limited

- According to GWAS data, the effect of obesity-associated variants correlates with the effect of type 2 diabetes, indicating a general effect of these loci on type 2 diabetes

- The obesity-associated variants display both metabolically healthy and unhealthy secondary associations

- Ongoing studies are seeking to elucidate the impact of rare variants in the pathogenesis of type 2 diabetes and obesity

\section{Future directions}

There have recently been dramatic changes to the technological and conceptual approaches used in genetic research of complex traits, leading to great advances. GWASs have been efficient in identifying common risk variants for metabolic disease, yet major efforts are still needed to gain biological knowledge from discoveries.

The search for genetic risk factors for type 2 diabetes and obesity is now targeting low-frequency and rare variations, and combining major data sets will presumably enable discoveries within the coming years, at first primarily focused on coding variation. However, we are on the path towards new technological and methodological developments, which will allow for large-scale genome sequencing [143]. Such developments will take genome sequencing to the population scale, possibly leading to a new understanding of the composite genetic architecture of human complex traits, integrating diverse kinds of genetic variation. For example, the study of copy number variations has led to important findings of association with severe obesity [122]. Furthermore, systemic integration of complex data obtained from other 'omics' techniques such as transcriptomics, proteomics and metabolomics and modelling of the combined composite impact of common metabolic phenotypes is projected to lead to breakthroughs in understanding the genetic determinants of metabolic traits.

As discussed above, common genetic variants unanimously impose modest risk increments on type 2 diabetes and adiposity. Furthermore, combining these variants does not enable prediction of type 2 diabetes [144, 145] or obesity $[45,146]$. Discovered variants explain a modest part of the heritability of metabolic diseases and future studies may reveal further important genetic susceptibility elements. Besides gaining biological knowledge and allowing the identification of at-risk individuals, hopes have been high that a knowledge of genetic risk factors would lead to personalised treatment based on the genetic profile. While detailed, sufficiently statistically powered studies of the genetic influence on treatment outcomes are still lacking, lessons from monogenic metabolic disease suggest that the identification of genetically homogenous groups may lead to improvements in individualised treatment [147]. In terms of the extent to which treatment can be individualised based on genetic information, it is possible that this will range from monogenic subsets, for whom highly individualised treatment can be provided, to genetic identification of pathophysiological specific subgroups of patients for whom stratified treatment can be given, and, finally, to a highly heterogeneous patient group, for which genetic profile knowledge will not add significantly to clinical care. Yet, with the ever-falling costs of genome sequencing, we will reach a situation in the future where all patients have their full genome sequenced, thereby allowing large-scale and more accurate studies of the genetic impact on treatment outcome in different patient strata.

Acknowledgements We thank the DIAbetes Genetics Replication and Meta-analysis (DIAGRAM) consortium (http://diagram-consortium.org/), the Meta-Analyses of Glucose and Insulin-related traits Consortium (MAGIC, http://www.magicinvestigators.org/) and the Genetic Investigation of ANthropometric Traits (GIANT) consortium (http:// www.broadinstitute.org/collaboration/giant/) consortia for summary results from genome-wide association studies. Data from the GIANT consortium were kindly provided by T. Frayling (University of Exeter, Exeter, UK) and I. Heid (University of Regensburg, Regensburg, Germany). Furthermore, we wish to thank staff at the University of Copenhagen, Copenhagen, Denmark, A. L. Nielsen and G. Lademann for management assistance.

Funding Our research is supported by The Lundbeck Foundation Centre for Applied Medical Genomics in Personalised Disease Prediction, Prevention, and Care (www.lucamp.org). The Novo Nordisk Foundation Center for Basic Metabolic Research is an independent Research Center at the University of Copenhagen partially funded by an unrestricted donation from the Novo Nordisk Foundation (www.metabol.ku.dk).

Contribution statement All authors were responsible for the conception and design of the manuscript, drafting the article and revising it critically for intellectual content. All authors approved the version to be published.

Duality of interest The authors declare that there is no duality of interest associated with this manuscript.

\section{References}

1. Kelly T, Yang W, Chen CS, Reynolds K, He J (2008) Global burden of obesity in 2005 and projections to 2030. Int J Obes (Lond) 32: 1431-1437 
2. Whiting DR, Guariguata L, Weil C, Shaw J (2011) IDF diabetes atlas: global estimates of the prevalence of diabetes for 2011 and 2030. Diabetes Res Clin Pract 94:311-321

3. Park Y, Zhu S, Palaniappan L, Heshka S, Carnethon MR, Heymsfield SB (2003) The metabolic syndrome: prevalence and associated risk factor findings in the US population from the Third National Health and Nutrition Examination Survey, 1988-1994. Arch Intern Med 163:427-436

4. Maier W, Holle R, Hunger M et al (2013) The impact of regional deprivation and individual socio-economic status on the prevalence of type 2 diabetes in Germany. A pooled analysis of five populationbased studies. Diabet Med 30:e78-e86

5. Rathmann W, Scheidt-Nave C, Roden M, Herder C (2013) Type 2 diabetes: prevalence and relevance of genetic and acquired factors for its prediction. Dtsch Arztebl Int 110:331-337

6. Speakman JR, O'Rahilly S (2012) Fat: an evolving issue. Dis Model Mech 5:569-573

7. Stunkard AJ, Harris JR, Pedersen NL, McClearn GE (1990) The body-mass index of twins who have been reared apart. N Engl J Med 322:1483-1487

8. Vogler GP, Sorensen TI, Stunkard AJ, Srinivasan MR, Rao DC (1995) Influences of genes and shared family environment on adult body mass index assessed in an adoption study by a comprehensive path model. Int J Obes Relat Metab Disord 19:40-45

9. Köbberling J (1982) The predictive value of diagnostic measures. Dtsch Med Wochenschr 107:591-595 (Article in German)

10. Almgren P, Lehtovirta M, Isomaa B et al (2011) Heritability and familiality of type 2 diabetes and related quantitative traits in the Botnia Study. Diabetologia 54:2811-2819

11. Zeggini E, Scott LJ, Saxena R et al (2008) Meta-analysis of genome-wide association data and large-scale replication identifies additional susceptibility loci for type 2 diabetes. Nat Genet 40:638645

12. Voight BF, Scott LJ, Steinthorsdottir V et al (2010) Twelve type 2 diabetes susceptibility loci identified through large-scale association analysis. Nat Genet 42:579-589

13. Morris AP, Voight BF, Teslovich TM et al (2012) Large-scale association analysis provides insights into the genetic architecture and pathophysiology of type 2 diabetes. Nat Genet 44:981-990

14. Albrechtsen A, Grarup N, Li Y et al (2013) Exome sequencingdriven discovery of coding polymorphisms associated with common metabolic phenotypes. Diabetologia 56:298-310

15. Steinthorsdottir V, Thorleifsson G, Sulem P et al (2014) Identification of low-frequency and rare sequence variants associated with elevated or reduced risk of type 2 diabetes. Nat Genet 46 : 294-298

16. Dupuis J, Langenberg C, Prokopenko I et al (2010) New genetic loci implicated in fasting glucose homeostasis and their impact on type 2 diabetes risk. Nat Genet 42:105-116

17. Yamauchi T, Hara K, Maeda S et al (2010) A genome-wide association study in the Japanese population identifies susceptibility loci for type 2 diabetes at UBE2E2 and C2CD4A-C2CD4B. Nat Genet 42:864-868

18. Saxena R, Saleheen D, Been LF et al (2013) Genome-wide association study identifies a novel locus contributing to type 2 diabetes susceptibility in Sikhs of Punjabi origin from India. Diabetes 62: $1746-1755$

19. Tabassum R, Chauhan G, Dwivedi OP et al (2013) Genome-wide association study for type 2 diabetes in Indians identifies a new susceptibility locus at 2q21. Diabetes 62:977-986

20. Kooner JS, Saleheen D, Sim X et al (2011) Genome-wide association study in individuals of South Asian ancestry identifies six new type 2 diabetes susceptibility loci. Nat Genet 43:984-989

21. Li H, Gan W, Lu L et al (2013) A genome-wide association study identifies GRK5 and RASGRP1 as type 2 diabetes loci in Chinese Hans. Diabetes 62:291-298
22. Cho YS, Chen CH, Hu C et al (2012) Meta-analysis of genomewide association studies identifies eight new loci for type 2 diabetes in east Asians. Nat Genet 44:67-72

23. Saxena R, Elbers CC, Guo Y et al (2012) Large-scale gene-centric meta-analysis across 39 studies identifies type 2 diabetes loci. Am J Hum Genet 90:410-425

24. Hara K, Fujita H, Johnson TA et al (2014) Genome-wide association study identifies three novel loci for type 2 diabetes. Hum Mol Genet 23:239-246

25. DIAbetes Genetics Replication And Meta-analysis (DIAGRAM) consortium, Asian Genetic Epidemiology Network Type 2 Diabetes (AGEN-T2D) consortium, South Asian Type 2 Diabetes (SAT2D) consortium et al (2014) Genome-wide trans-ancestry meta-analysis provides insight into the genetic architecture of type 2 diabetes susceptibility. Nat Genet 46:234-244

26. Grant SF, Thorleifsson G, Reynisdottir I et al (2006) Variant of transcription factor 7-like 2 (TCF7L2) gene confers risk of type 2 diabetes. Nat Genet 38:320-323

27. Cauchi S, Achhab Y, Choquet H et al (2007) TCF7L2 is reproducibly associated with type 2 diabetes in various ethnic groups: a global meta-analysis. J Mol Med 85:777-782

28. Saxena R, Hivert MF, Langenberg C et al (2010) Genetic variation in GIPR influences the glucose and insulin responses to an oral glucose challenge. Nat Genet 42:142-148

29. Scott RA, Lagou V, Welch RP et al (2012) Large-scale association analyses identify new loci influencing glycemic traits and provide insight into the underlying biological pathways. Nat Genet 44:991-1005

30. Manning AK, Hivert MF, Scott RA et al (2012) A genome-wide approach accounting for body mass index identifies genetic variants influencing fasting glycemic traits and insulin resistance. Nat Genet 44:659-669

31. Soranzo N, Sanna S, Wheeler E et al (2010) Common variants at 10 genomic loci influence hemoglobin $\mathrm{A}_{1 \mathrm{C}}$ levels via glycemic and nonglycemic pathways. Diabetes 59:3229-3239

32. Müssig K, Staiger H, Machicao F, Haring HU, Fritsche A (2010) Genetic variants affecting incretin sensitivity and incretin secretion. Diabetologia 53:2289-2297

33. Grarup N, Sparsø T, Hansen T (2010) Physiologic characterization of type 2 diabetes-related loci. Curr Diab Rep 10:485-497

34. Rosengren AH, Braun M, Mahdi T et al (2012) Reduced insulin exocytosis in human pancreatic beta-cells with gene variants linked to type 2 diabetes. Diabetes 61:1726-1733

35. Boesgaard TW, Grarup N, Jørgensen T et al (2010) Variants at DGKB/TMEM195, ADRA2A, GLIS3 and C2CD4B loci are associated with reduced glucose-stimulated beta cell function in middleaged Danish people. Diabetologia 53:1647-1655

36. Ingelsson E, Langenberg C, Hivert MF et al (2010) Detailed physiologic characterization reveals diverse mechanisms for novel genetic loci regulating glucose and insulin metabolism in humans. Diabetes 59:1266-1275

37. Lyssenko V, Lupi R, Marchetti P et al (2007) Mechanisms by which common variants in the TCF7L2 gene increase risk of type 2 diabetes. J Clin Invest 117:2155-2163

38. Simonis-Bik AM, Nijpels G, van Haeften TW et al (2010) Gene variants in the novel type 2 diabetes loci CDC123/CAMK1D, THADA, ADAMTS9, BCL11A, and MTNR1B affect different aspects of pancreatic beta-cell function. Diabetes 59:293-301

39. Ferrannini E, Mari A (2004) Beta cell function and its relation to insulin action in humans: a critical appraisal. Diabetologia 47:943956

40. Rung J, Cauchi S, Albrechtsen A et al (2009) Genetic variant near IRS1 is associated with type 2 diabetes, insulin resistance and hyperinsulinemia. Nat Genet 41:1110-1115

41. Frayling TM, Timpson NJ, Weedon MN et al (2007) A common variant in the FTO gene is associated with body mass 
index and predisposes to childhood and adult obesity. Science 316:889-894

42. Loos RJ, Lindgren CM, Li S et al (2008) Common variants near $\mathrm{MC} 4 \mathrm{R}$ are associated with fat mass, weight and risk of obesity. Nat Genet 40:768-775

43. Thorleifsson G, Walters GB, Gudbjartsson DF et al (2009) Genomewide association yields new sequence variants at seven loci that associate with measures of obesity. Nat Genet 41:18-24

44. Willer CJ, Speliotes EK, Loos RJ et al (2009) Six new loci associated with body mass index highlight a neuronal influence on body weight regulation. Nat Genet 41:25-34

45. Speliotes EK, Willer CJ, Berndt SI et al (2010) Association analyses of 249,796 individuals reveal 18 new loci associated with body mass index. Nat Genet 42:937-948

46. Chambers JC, Elliott P, Zabaneh D et al (2008) Common genetic variation near MC4R is associated with waist circumference and insulin resistance. Nat Genet 40:716-718

47. Lindgren CM, Heid IM, Randall JC et al (2009) Genome-wide association scan meta-analysis identifies three Loci influencing adiposity and fat distribution. PLoS Genet 5:e1000508

48. Heid IM, Jackson AU, Randall JC et al (2010) Meta-analysis identifies 13 new loci associated with waist-hip ratio and reveals sexual dimorphism in the genetic basis of fat distribution. Nat Genet 42:949-960

49. Meyre D, Delplanque J, Chèvre JC et al (2009) Genome-wide association study for early-onset and morbid adult obesity identifies three new risk loci in European populations. Nat Genet 41:157-159

50. Scherag A, Dina C, Hinney A et al (2010) Two new loci for bodyweight regulation identified in a joint analysis of genome-wide association studies for early-onset extreme obesity in French and German Study Groups. PLoS Genet 6:e1000916

51. Bradfield JP, Taal HR, Timpson NJ et al (2012) A genome-wide association meta-analysis identifies new childhood obesity loci. Nat Genet 44:526-531

52. Jiao H, Arner P, Hoffstedt J et al (2011) Genome wide association study identifies KCNMA1 contributing to human obesity. BMC Med Genom 4:51

53. Paternoster L, Evans DM, Nohr EA et al (2011) Genome-wide population-based association study of extremely overweight young adults-the GOYA study. PLoS One 6:e24303

54. Wheeler E, Huang N, Bochukova EG et al (2013) Genome-wide SNP and CNV analysis identifies common and low-frequency variants associated with severe early-onset obesity. Nat Genet 45:513-517

55. Berndt SI, Gustafsson S, Magi R et al (2013) Genome-wide metaanalysis identifies 11 new loci for anthropometric traits and provides insights into genetic architecture. Nat Genet 45:501-512

56. Mitchell JA, Hakonarson H, Rebbeck TR, Grant SF (2013) Obesitysusceptibility loci and the tails of the pediatric BMI distribution. Obesity (Silver Spring) 21:1256-1260

57. Yang J, Loos RJ, Powell JE et al (2012) FTO genotype is associated with phenotypic variability of body mass index. Nature 490:267272

58. Unoki H, Takahashi A, Kawaguchi T et al (2008) SNPs in KCNQ1 are associated with susceptibility to type 2 diabetes in East Asian and European populations. Nat Genet 40: 1098-1102

59. Yasuda K, Miyake K, Horikawa Y et al (2008) Variants in KCNQ1 are associated with susceptibility to type 2 diabetes mellitus. Nat Genet 40:1092-1097

60. Wen W, Cho YS, Zheng W et al (2012) Meta-analysis identifies common variants associated with body mass index in east Asians. Nat Genet 44:307-311

61. Monda KL, Chen GK, Taylor KC et al (2013) A meta-analysis identifies new loci associated with body mass index in individuals of African ancestry. Nat Genet 45:690-696
62. Waters KM, Stram DO, Hassanein MT et al (2010) Consistent association of type 2 diabetes risk variants found in Europeans in diverse racial and ethnic groups. PLoS Genet 6:e1001078

63. Haiman CA, Fesinmeyer MD, Spencer KL et al (2012) consistent directions of effect for established type 2 diabetes risk variants across populations: The Population Architecture using Genomics and Epidemiology (PAGE) Consortium. Diabetes 61:1642-1647

64. Okada Y, Kubo M, Ohmiya H et al (2012) Common variants at CDKAL1 and KLF9 are associated with body mass index in east Asian populations. Nat Genet 44:302-306

65. Diabetes Genetics Initiative of Broad Institute of Harvard and MIT, Lund University and Novartis Institutes of BioMedical Research, Saxena R, Voight BF et al (2007) Genome-wide association analysis identifies loci for type 2 diabetes and triglyceride levels. Science 316:1331-1336

66. Beer NL, Tribble ND, McCulloch LJ et al (2009) The P446L variant in GCKR associated with fasting plasma glucose and triglyceride levels exerts its effect through increased glucokinase activity in liver. Hum Mol Genet 18:4081-4088

67. Rees MG, Wincovitch S, Schultz J et al (2012) Cellular characterisation of the GCKR P446L variant associated with type 2 diabetes risk. Diabetologia 55:114-122

68. Helgason A, Palsson S, Thorleifsson G et al (2007) Refining the impact of TCF7L2 gene variants on type 2 diabetes and adaptive evolution. Nat Genet 39:218-225

69. Zhou Y, Zhang E, Berggreen C et al (2012) Survival of pancreatic beta cells is partly controlled by a TCF7L2-p53-p53INP1-dependent pathway. Hum Mol Genet 21:196-207

70. Mondal AK, Das SK, Baldini G et al (2010) Genotype and tissuespecific effects on alternative splicing of the transcription factor 7 like 2 gene in humans. J Clin Endocrinol Metab 95:1450-1457

71. Gaulton KJ, Nammo T, Pasquali L et al (2010) A map of open chromatin in human pancreatic islets. Nat Genet 42:255-259

72. Froguel P, Vaxillaire M, Sun F et al (1992) Close linkage of glucokinase locus on chromosome $7 \mathrm{p}$ to early-onset non-insulindependent diabetes mellitus. Nature 356:162-164

73. Horikawa Y, Iwasaki N, Hara M et al (1997) Mutation in hepatocyte nuclear factor-1 beta gene (TCF2) associated with MODY. Nat Genet 17:384-385

74. Yamagata K, Oda N, Kaisaki PJ et al (1996) Mutations in the hepatocyte nuclear factor- $1 \alpha$ gene in maturity-onset diabetes of the young (MODY3). Nature 384:455-458

75. Yamagata K, Furuta H, Oda N et al (1996) Mutations in the hepatocyte nuclear factor- $4 \alpha$ gene in maturity-onset diabetes of the young (MODY1). Nature 384:458-460

76. Barroso I, Gurnell M, Crowley VE et al (1999) Dominant negative mutations in human PPAR $\gamma$ associated with severe insulin resistance, diabetes mellitus and hypertension. Nature 402:880-883

77. Gloyn AL (2004) Activating mutations in the gene encoding the ATP-sensitive potassium-channel subunit Kir6.2 and permanent neonatal diabetes. N Engl J Med 350:1838-1849

78. Senee V, Chelala C, Duchatelet S et al (2006) Mutations in GLIS3 are responsible for a rare syndrome with neonatal diabetes mellitus and congenital hypothyroidism. Nat Genet 38:682-687

79. Inoue H, Tanizawa Y, Wasson J et al (1998) A gene encoding a transmembrane protein is mutated in patients with diabetes mellitus and optic atrophy (Wolfram syndrome). Nat Genet 20:143-148

80. Gudmundsson J, Sulem P, Steinthorsdottir V et al (2007) Two variants on chromosome 17 confer prostate cancer risk, and the one in TCF2 protects against type 2 diabetes. Nat Genet 39: 977-983

81. Krude H, Biebermann H, Luck W, Horn R, Brabant G, Grüters A (1998) Severe early-onset obesity, adrenal insufficiency and red hair pigmentation caused by POMC mutations in humans. Nat Genet 19: $155-157$ 
82. Yeo GS, Farooqi IS, Aminian S, Halsall DJ, Stanhope RG, O'Rahilly S (1998) A frameshift mutation in MC4R associated with dominantly inherited human obesity. Nat Genet 20:111-112

83. Clement K, Vaisse C, Lahlou N et al (1998) A mutation in the human leptin receptor gene causes obesity and pituitary dysfunction. Nature 392:398-401

84. Han JC, Liu QR, Jones M et al (2008) Brain-derived neurotrophic factor and obesity in the WAGR syndrome. N Engl J Med 359:918927

85. Bochukova EG, Huang N, Keogh J et al (2010) Large, rare chromosomal deletions associated with severe early-onset obesity. Nature 463:666-670

86. Jackson RS, Creemers JW, Ohagi S et al (1997) Obesity and impaired prohormone processing associated with mutations in the human prohormone convertase 1 gene. Nat Genet 16: 303-306

87. Yeo GS, Connie Hung CC, Rochford J et al (2004) A de novo mutation affecting human TrkB associated with severe obesity and developmental delay. Nat Neurosci 7:1187-1189

88. Scuteri A, Sanna S, Chen WM et al (2007) Genome-wide association scan shows genetic variants in the FTO gene are associated with obesity-related traits. PLoS Genet 3:e115

89. Dina C, Meyre D, Gallina S et al (2007) Variation in FTO contributes to childhood obesity and severe adult obesity. Nat Genet 39: 724-726

90. Hertel JK, Johansson S, Sonestedt E et al (2011) FTO, type 2 diabetes, and weight gain throughout adult life: a meta-analysis of 41,504 subjects from the Scandinavian HUNT, MDC, and MPP studies. Diabetes 60:1637-1644

91. Li H, Kilpelainen TO, Liu C et al (2012) Association of genetic variation in FTO with risk of obesity and type 2 diabetes with data from 96,551 East and South Asians. Diabetologia 55:981-995

92. Meyre D (2012) Is FTO a type 2 diabetes susceptibility gene? Diabetologia 55:873-876

93. Gardner KM, Latta RG (2007) Shared quantitative trait loci underlying the genetic correlation between continuous traits. Mol Ecol 16:4195-4209

94. Carlsson S, Ahlbom A, Lichtenstein P, Andersson T (2013) Shared genetic influence of BMI, physical activity and type 2 diabetes: a twin study. Diabetologia 56:1031-1035

95. Lehtovirta M, Pietiläinen KH, Levälahti E et al (2010) Evidence that BMI and type 2 diabetes share only a minor fraction of genetic variance: a follow-up study of 23,585 monozygotic and dizygotic twins from the Finnish Twin Cohort Study. Diabetologia 53:1314 1321

96. Teslovich TM, Musunuru K, Smith AV et al (2010) Biological, clinical and population relevance of 95 loci for blood lipids. Nature 466:707-713

97. van Vliet-Ostaptchouk JV, den Hoed M, Luan J et al (2013) Pleiotropic effects of obesity-susceptibility loci on metabolic traits: a meta-analysis of up to 37,874 individuals. Diabetologia $56: 2134$ 2146

98. Cooney GJ, Lyons RJ, Crew AJ et al (2004) Improved glucose homeostasis and enhanced insulin signalling in Grb14-deficient mice. EMBO J 23:582-593

99. Robiou-du-Pont S, Bonnefond A, Yengo L et al (2013) Contribution of 24 obesity-associated genetic variants to insulin resistance, pancreatic beta-cell function and type 2 diabetes risk in the French population. Int J Obes (Lond) 37:980-985

100. Kilpelainen TO, Zillikens MC, Stancakova A et al (2011) Genetic variation near IRS1 associates with reduced adiposity and an impaired metabolic profile. Nat Genet 43:753-760

101. Thomsen M, Nordestgaard BG (2014) Myocardial infarction and ischemic heart disease in overweight and obesity with and without metabolic syndrome. JAMA Intern Med 174:15-22
102. Kramer CK, Zinman B, Retnakaran R (2013) Are metabolically healthy overweight and obesity benign conditions? A systematic review and meta-analysis. Ann Intern Med 159:758-769

103. Maher B (2008) Personal genomes: the case of the missing heritability. Nature 456:18-21

104. Reich DE, Lander ES (2001) On the allelic spectrum of human disease. Trends Genet 17:502-510

105. Pritchard JK, Cox NJ (2002) The allelic architecture of human disease genes: common disease-common variant...or not? Hum Mol Genet 11:2417-2423

106. Bodmer W, Bonilla C (2008) Common and rare variants in multifactorial susceptibility to common diseases. Nat Genet 40:695-701

107. Pritchard JK (2001) Are rare variants responsible for susceptibility to complex diseases? Am J Hum Genet 69:124-137

108. 1000 Genomes Project Consortium, Durbin RM, Abecasis GR et al (2010) A map of human genome variation from population-scale sequencing. Nature 467:1061-1073

109. Tennessen JA, Bigham AW, O'Connor TD et al (2012) Evolution and functional impact of rare coding variation from deep sequencing of human exomes. Science 337:64-69

110. Barton NH, Turelli M (1989) Evolutionary quantitative genetics: how little do we know? Annu Rev Genet 23:337-370

111. Neel JV (1962) Diabetes mellitus: a "thrifty" genotype rendered detrimental by "progress"? Am J Hum Genet 14:353-362

112. Ayub Q, Moutsianas L, Chen Y et al (2014) Revisiting the thrifty gene hypothesis via 65 loci associated with susceptibility to type 2 diabetes. Am J Hum Genet 94:176-185

113. Cohen JC, Kiss RS, Pertsemlidis A, Marcel YL, McPherson R, Hobbs HH (2004) Multiple rare alleles contribute to low plasma levels of HDL cholesterol. Science 305:869-872

114. Larsen LH, Echwald SM, Sorensen TI, Andersen T, Wulff BS, Pedersen O (2005) Prevalence of mutations and functional analyses of melanocortin 4 receptor variants identified among 750 men with juvenile-onset obesity. J Clin Endocrinol Metab 90:219-224

115. Creemers JW, Choquet H, Stijnen P et al (2012) Heterozygous mutations causing partial prohormone convertase 1 deficiency contribute to human obesity. Diabetes 61:383-390

116. Ichimura A, Hirasawa A, Poulain-Godefroy O et al (2012) Dysfunction of lipid sensor GPR120 leads to obesity in both mouse and human. Nature 483:350-354

117. Rivas MA, Beaudoin M, Gardet A et al (2011) Deep resequencing of GWAS loci identifies independent rare variants associated with inflammatory bowel disease. Nat Genet 43:1066-1073

118. Bonnefond A, Clement N, Fawcett K et al (2012) Rare MTNR1B variants impairing melatonin receptor $1 \mathrm{~B}$ function contribute to type 2 diabetes. Nat Genet 44:297-301

119. Visscher PM, Hill WG, Wray NR (2008) Heritability in the genomics era - concepts and misconceptions. Nat Rev Genet 9:255-266

120. Willer CJ, Schmidt EM, Sengupta S et al (2013) Discovery and refinement of loci associated with lipid levels. Nat Genet 45:1274 1283

121. Agarwala V, Flannick J, Sunyaev S, Altshuler D (2013) Evaluating empirical bounds on complex disease genetic architecture. Nat Genet 45:1418-1427

122. Walters RG, Jacquemont S, Valsesia A et al (2010) A new highly penetrant form of obesity due to deletions on chromosome 16p11.2. Nature 463:671-675

123. Andreasen $\mathrm{CH}$, Stender-Petersen KL, Mogensen MS et al (2008) Low physical activity accentuates the effect of the FTO rs9939609 polymorphism on body fat accumulation. Diabetes 57:95-101

124. Kilpelainen TO, Qi L, Brage S et al (2011) Physical activity attenuates the influence of FTO variants on obesity risk: a metaanalysis of 218,166 adults and 19,268 children. PLoS Med 8: e1001116 
125. Kong A, Steinthorsdottir V, Masson G et al (2009) Parental origin of sequence variants associated with complex diseases. Nature 462 : 868-874

126. Zuk O, Schaffner SF, Samocha K et al (2014) Searching for missing heritability: designing rare variant association studies. Proc Natl Acad Sci 111:E455-E464

127. Holm H, Gudbjartsson DF, Sulem P et al (2011) A rare variant in MYH6 is associated with high risk of sick sinus syndrome. Nat Genet 43:316-320

128. Wood AR, Perry JRB, Tanaka T et al (2013) Imputation of variants from the 1000 Genomes Project modestly improves known associations and can identify low-frequency variant - phenotype associations undetected by HapMap based imputation. PLoS ONE 8: e64343

129. Zheng HF, Ladouceur M, Greenwood CMT, Richards JB (2012) Effect of genome-wide genotyping and reference panels on rare variants imputation. J Genet Genomics 39:545-550

130. Grarup N, Sulem P, Sandholt CH et al (2013) Genetic architecture of vitamin B12 and folate levels uncovered applying deeply sequenced large datasets. PLoS Genet 9:e1003530

131. Heinzen EL, Depondt C, Cavalleri GL et al (2012) Exome sequencing followed by large-scale genotyping fails to identify single rare variants of large effect in idiopathic generalized epilepsy. Am J Hum Genet 91:293-302

132. Need AC, McEvoy JP, Gennarelli M et al (2012) Exome sequencing followed by large-scale genotyping suggests a limited role for moderately rare risk factors of strong effect in schizophrenia. Am J Hum Genet 91:303-312

133. Huyghe JR, Jackson AU, Fogarty MP et al (2013) Exome array analysis identifies new loci and low-frequency variants influencing insulin processing and secretion. Nat Genet 45: 197-201

134. Stitziel NO, Kiezun A, Sunyaev S (2011) Computational and statistical approaches to analyzing variants identified by exome sequencing. Genome Biol 12:227
135. Ladouceur M, Dastani Z, Aulchenko YS, Greenwood CM, Richards JB (2012) The empirical power of rare variant association methods: results from sanger sequencing in 1,998 individuals. PLoS Genet 8: e1002496

136. Lohmueller KE, Sparsø T, Li Q et al (2013) Whole-exome sequencing of 2,000 Danish individuals and the role of rare coding variants in type 2 diabetes. Am J Hum Genet 93:1072-1086

137. Saad M, Wijsman EM (2014) Power of family-based association designs to detect rare variants in large pedigrees using imputed genotypes. Genet Epidemiol 38:1-9

138. Risch N, Merikangas K (1996) The future of genetic studies of complex human diseases. Science 273:1516-1517

139. Sebat J, Lakshmi B, Malhotra D et al (2007) Strong association of de novo copy number mutations with autism. Science 316:445-449

140. Neale BM, Kou Y, Liu L et al (2012) Patterns and rates of exonic de novo mutations in autism spectrum disorders. Nature 485:242-245

141. Kong A, Frigge ML, Masson G et al (2012) Rate of de novo mutations and the importance of father's age to disease risk. Nature 488:471-475

142. Bellanné-Chantelot C, Chauveau D, Gautier JF et al (2004) Clinical spectrum associated with hepatocyte nuclear factor- $1 \beta$ mutations. Ann Intern Med 140:510-517

143. Schadt EE, Turner S, Kasarskis A (2010) A window into thirdgeneration sequencing. Hum Mol Genet 19:R227-R240

144. Meigs JB, Shrader P, Sullivan LM et al (2008) Genotype score in addition to common risk factors for prediction of type 2 diabetes. N Engl J Med 359:2208-2219

145. Lyssenko V, Jonsson A, Almgren P et al (2008) Clinical risk factors, DNA variants, and the development of type 2 diabetes. N Engl J Med 359:2220-2232

146. Sandholt CH, Sparso T, Grarup N et al (2010) Combined analyses of 20 common obesity susceptibility variants. Diabetes 59:1667-1673

147. Thanabalasingham G, Owen KR (2011) Diagnosis and management of maturity onset diabetes of the young (MODY). BMJ 343:d6044 